\title{
Measurement of lung volumes by plethysmography
}

\author{
A.L. Coates*, R. Peslin**, D. Rodenstein + , J. Stocks ${ }^{++}$
}

\section{CONTENTS}

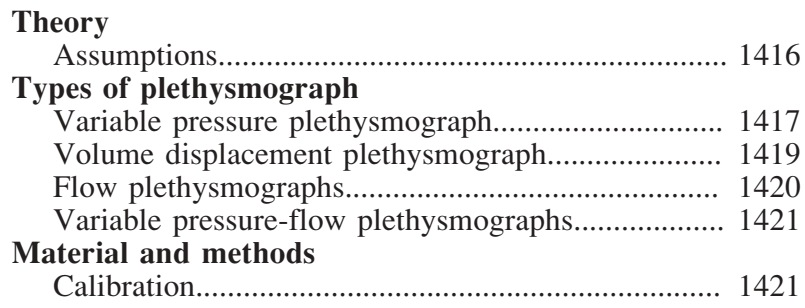

There are various ways of measuring absolute lung volume. These range from measurements derived from chest radiographs to the more common laboratory measurements employing either gas dilution or plethysmography. The principal difference between the latter two methods is that gas dilution techniques measure gas that is in free communication with the airway opening, whilst plethysmography measures all intrathoracic gas. Once the absolute lung volume is known, the other lung volumes can be measured from the change in volume during specific respiratory manoeuvres.

Work on this article was undertaken as part of a joint European Respiratory Society/American Thoracic Society effort to develop a joint "Statement" on the measurement of lung volumes. It will review the principles, practice and limitations of plethysmography, and recommend standards for the plethysmographic measure of lung volumes. These standards will include equipment specifications and measurement techniques over the age range from infancy to adulthood.

For the purpose of this review, functional residual capacity (FRC) is defined as the absolute volume of gas in the lung at the end of a normal expiration. Thoracic gas volume (TGV) is defined as the volume of intrathoracic gas at the time the airway is occluded for the plethysmographic measurement; while this is usually at $\mathrm{FRC}$, in special circumstances it may not be $[1,2]$. Irrespective of where in the volume cycle TGV is measured, it should be adjusted to the FRC derived from

\begin{tabular}{|c|c|}
\hline \multicolumn{2}{|l|}{ Thermal drift } \\
\hline Frequency response............. & 1422 \\
\hline Measurement techniques.. & 1423 \\
\hline Quality control... & 1424 \\
\hline Plethysmograph in infants & \\
\hline Measurement techniques... & 142 \\
\hline 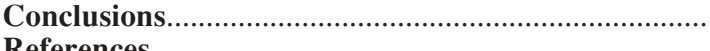 & 142 \\
\hline
\end{tabular}

plethysmography (FRCpleth) by subtracting or adding the appropriate volume correction. In normal children and adults, there should be no difference in FRC between gas dilution techniques and plethysmography. However, in patients with lung disease associated with gas-trapping and in normal infants [3], FRCpleth generally exceeds FRC measured by gas dilution.

\section{Theory}

In 1956, DuBois et al. [4] described a technique for measuring the volume of gas in the thorax by using the measurement of a volume change when a gas is compressed, as assessed by Boyle's Law. Boyle's Law states that, under isothermal conditions, when gas in a closed container is compressed, its volume decreases while the pressure inside the container increases, such that the product of volume and pressure, at any given moment, is constant. Isothermal conditions imply that, during either compression or rarefaction, heat is exchanged across the walls of the container so that the temperature of the gas remains the same. If no heat is lost, changes in gas temperature occur and conditions are considered to be adiabatic and follow the Ideal Gas Law. This states that the product of pressure $(P)$ and volume $(V)$ divided by temperature remains constant. Conditions in between these two extremes are polytropic. For plethysmography, Boyle's Law is:

\footnotetext{
*Dept of Pediatrics, McGill University, Faculty of Medicine, Montréal, Québec, Canada. **INSERM U14, Vandoeuvre les Nancy, France. +Université Catholique de Louvin, Clinical Universitaires Saint-Luc, Bruxelles, Belgium. ${ }^{++}$Portex Anaesthesia, Intensive Care and Respiratory Medicine Unit, Institute of Child Health, London, UK.

Correspondence: A.L. Coates, The Montreal Children's Hospital, Division of Respiratory Medicine, Room D-380, 230 Tupper Street, Montréal, Québec, H3H 1P3, Canada

Keywords: Infant pulmonary function test, lung volumes, plethysmography

Received: June 24 1996; Accepted after revision August 191996

This publication evolved from a workshop on "measurement of lung volumes" convened by the European Respiratory Society and the American Thoracic Society, with additional support from the National Heart, Lung and Blood Institute (Grant No. R13 HL48384).
} 


$$
P_{1} \times V_{1}=P_{2} \times V_{2}
$$

where the smaller script "1" and "2" denote the first and second condition of the gas. Applied to the determination of TGV, the subject is placed in an enclosed space, the plethysmograph, and is instructed to pant against an obstruction at the airway opening at a frequency of approximately $1 \mathrm{~Hz}$ [5-7], resulting in the rarefaction and compression of the gas in the thorax. During these manoeuvres, changes in thoracic volume (measured from the plethysmograph) and pressure measured at the airway opening $(P$ ao $)$ are recorded. Assuming that $P$ ao is representative of alveolar pressure $(P \mathrm{~A})$, TGV can be calculated using the following application of Boyle's Law:

$$
\left(P \mathrm{~A} 1-P_{2} \mathrm{O}\right) \times \mathrm{TGV} 1=\left(P \mathrm{~A} 2-P \mathrm{H}_{2} \mathrm{O}\right) \times \mathrm{TGV} 2
$$

where the smaller script denotes differing values of pressure and volume during the respiratory manoeuvre. $P$ A1 and $P$ A2 are expressed as absolute pressures, not the differences between barometric pressure $(P B)$ and $P$ ao. $P \mathrm{H}_{2} \mathrm{O}$ represents water vapour pressure of saturated gas at $37^{\circ} \mathrm{C}(6.3 \mathrm{kPa})$. Water vapour pressure is subtracted from all pressures because, in saturated gas, its magnitude relates only to temperature, passing in and out of the vapour state as the total pressure changes. Hence, under fully saturated conditions, water vapour does not behave as a compressible gas. For the sake of clarity in the development of this theoretical basis, $\mathrm{PH}_{2} \mathrm{O}$ will not appear in the subsequent equations, but must be subtracted from all measurements of $P$ A or $P B$.

A more common way to write Equation (2) is:

$$
P A 1 \times \mathrm{TGV}_{1}=(P \mathrm{~A} 1+\Delta P) \times(\mathrm{TGV} 1+\Delta V)
$$

which, when all term are multiplied becomes:

$$
\begin{aligned}
P \mathrm{~A} 1 \times \mathrm{TGV} 1= & P \mathrm{~A} 1 \times \mathrm{TGV}_{1}+\Delta V \times P \mathrm{~A} 1+\Delta P \times \mathrm{TGV}_{1}+\Delta P \times \Delta V \\
& \text { or }-\Delta P \times \mathrm{TGV}_{1}=\Delta V(P \mathrm{~A} 1+\Delta P)
\end{aligned}
$$

where $P$ A1 is the alveolar pressure at the start of the manoeuvre, and $\Delta P$ is the change in the alveolar pressure measured at the airway opening under conditions of no flow during the panting manoeuvre, so that $\Delta P$ $=P$ A2 $-P$ A1.$\Delta V$ is the resulting change in volume of the thorax, and is equal to TGV2 - TGV1.

Solving Equation (3) for TGV1:

$$
\mathrm{TGV}_{1}=-(\Delta V / \Delta P) \times(P \mathrm{~A} 1+\Delta P)
$$

Since $\Delta P=(P \mathrm{~A} 2-P \mathrm{~A} 1)$, this can be further simplified to:

$$
\mathrm{TGV}_{1}=-(\Delta V / \Delta P) \times P \mathrm{~A} 2
$$

When performing a panting manoeuvre, it is frequently assumed that the pressure changes are small $( \pm 1$ $\mathrm{kPa}$ ). Under these conditions, it is customary to ignore the small product of $\Delta P \times \Delta V$, the last term in Equation ( $3 \mathrm{a})$, and the solution becomes:

$$
\mathrm{TGV}_{1}=-(\Delta V / \Delta P) \times P \mathrm{~A} 1
$$

As noted previously, PA1 represents the alveolar pressure at the start of a panting manoeuvre, and it is generally assumed that the discrepancy between $P$ A1 and $P \mathrm{~B}$ during a panting manoeuvre is small so that the solution for $\mathrm{TGV} 1$ is reduced to:

$$
\mathrm{TGV}=-(\Delta V / \Delta P) \times P \mathrm{~B}
$$

The term $\Delta \mathrm{V} / \Delta \mathrm{P}$ is the slope of the pressure volume relationship and is always negative. While technically a hyperbola, it is so close to being a straight line over the range of interest, that few errors are introduced when it is treated as such. This "simplified" equation is the one used in most automated plethysmographs.

Because of the problems experienced with the panting technique, particularly the difficulty in obtaining even gentle pants at a frequency of $1 \mathrm{~Hz}$, a single inspiratory effort has been proposed as an alternative [8]. However, this method of determining TGV may give rise to changes in pressure that are of sufficient magnitude (i.e. up to $5 \mathrm{kPa}$ ) that the $\Delta P \times \Delta V$ should not be ignored [9]. An additional consideration when calculating $\mathrm{TGV}$ is that where $P \mathrm{~A} 1$ differs from $P \mathrm{~B}$, such as will occur if the occlusion is not performed at FRC, a correction factor $(P \mathrm{~A} 1 / P \mathrm{~B})$ may be needed to convert TGV to body temperature and pressure saturated (BTPS). Hence, Equation (3c) can be rewritten as:

$$
\mathrm{TGV}=-(\Delta V / \Delta P) \times P \mathrm{~A} 2 \times(P \mathrm{~A} 1 / P \mathrm{~B})
$$

This is the "complete" equation and it contains both the $\Delta P \times \Delta V$ term and volume correction necessary should $P$ A1 be different from $\mathrm{PB}$. As mentioned pre-

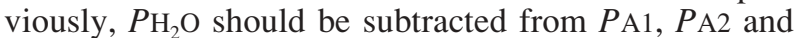
$P \mathrm{~B}$. With today's modern computing facilities, there is no reason to use the simplified version of Boyle's Law, Equation (5), rather than the complete version, Equation (6). The errors introduced by the simplified version during panting are small (in the order of 3\%) and centred around zero. For the single inspiratory manoeuvre, they are in the order of $5 \%$, systematically greater than zero and related to the magnitude of the pressure generated during the manoeuvre [9]. Furthermore, such systematic errors are unnecessary [9].

\section{Assumptions}

The plethysmographic method of determining FRCpleth is based on a number of assumptions, which are as follows:

1. There is no gas flow, and hence no flow-resistive losses of pressure in the airways, during the respiratory efforts against an occluded airway. Hence, $P$ ao is equal to $P$ A.

This assumption has been challenged $[10,11]$. In adults, it has been suggested that the upper airway, particularly the pharynx and cheeks, acts as a shunt capacitor, allowing gas flow back and forth in the airway during the panting manoeuvre. This would result in $\Delta P$ ao underestimating $\triangle P \mathrm{~A}$, leading to an overestimate of FRCpleth. While, theoretically, this problem could be a consideration in all subjects, only those with significant airway obstruction, either due to asthma [10] or artificially induced [11], have been shown to have high 
enough airway resistance to cause appreciable losses in pressure. Panting at slower frequencies (i.e. around 1 $\mathrm{Hz}$ ) may reduce, but not completely eliminate, the error $[6,7]$, possibly due to persistent intrapulmonary gas flow during the occlusion resulting from nonuniform alveolar pressures (see below). There are few data concerning flow resistive losses during FRCpleth measurements in infants and neonates, but work performed in older children with extensive disease [8] suggests that panting frequency around $1 \mathrm{~Hz}$ reduces the error, as in adults $[6,7]$.

2. The pulmonary parenchyma is either sufficiently elastic, or gas-containing spaces are freely in communication with each other, so that changes in pressure are uniform throughout all the gas-containing areas of the lung.

This assumption, while probably reasonable in most situations in adults, has been challenged in infants with airway disease. GODFREY et al. [12] found values of FRCpleth that were much lower than expected from clinical findings in infants recovering from bronchiolitis. By a process of elimination, they suggested that there must be areas in the lungs of these infants that have such high resistance and low compliance that they act as little "spheres", whose contents do not undergo volume changes during the panting manoeuvre. HeLMS [13] provided evidence that more accurate measurements of FRCpleth could be obtained in infants with cardiopulmonary disease by performing airway occlusions at the end of inspiration, rather than end-expiration. This was attributed to the overestimation of TGV at low lung volumes due to airway closure in this group, results which were analogous to those reported by BROwN and Slutsky [7] in adults. A study by Beardsmore et al. [14] also suggested that airway closure during tidal breathing might lead to inaccuracies in FRCpleth if measured at low lung volumes, although the potential influence of the cheeks and upper airways when artificially inflating the lungs, as in this study, was not considered.

An alternative explanation for discrepancies in FRCpleth is that changes in pleural pressure during panting are nonuniform. This is controversial in adults; some evidence supports it [7], other workers have found no evidence of it [15]. The suggestion of nonuniform distribution of pleural pressure changes in neonates $[14,16]$ has not been supported by more recent work [17]. This would suggest that the effects of nonuniform pleural pressure changes, if they exist, are likely to be small. Part of the problem with this argument is that there is no "gold standard", which would allow adequate comparison of values measured by different techniques [18], since the abnormalities are seen only in subjects who have gastrapping and, hence, would have unreliable estimates of FRC by gas dilution techniques.

3. Only gas in the thorax undergoes rarefaction and compression.

This assumes that the volume of gas in the gastrointestinal tract is insignificant, not compressed or both. In infants [12,13] and adults [7], this appears to be a valid assumption, since the changes in intra-abdominal pressure when panting around FRC or higher lung volumes are relatively small compared to changes in pressure at the airway opening. They are also unpredictable [7]. Theoretically, this might be a problem in the presence of either increased abdominal gas, as frequently occurs in cystic fibrosis, or significant changes in abdominal pressure when panting.

\section{Types of plethysmograph}

The changes in thoracic volume which accompany compression or decompression of the gas in the lungs during respiratory manoeuvres can be obtained using a body plethysmograph by measuring the changes in: 1) pressure within a constant volume chamber (variable pressure plethysmograph), 2) volume within a constant pressure chamber (volume displacement plethysmograph) [19] or; 3) airflow in and out of a constant pressure chamber (flow plethysmograph).

\section{Variable pressure plethysmograph}

The advantages of the variable pressure plethysmograph [4] (fig. 1) are simplicity and accuracy of the measurement of small changes in volume, as are seen during panting. The changes in lung volume during rarefaction and compression occur sufficiently slowly and in the environment of a very high surface area to volume ratio to ensure that any heat generated during the manoeuvre is immediately lost to the lung tissue, so that changes in pressure and volume within the lung are essentially isothermal [20]. However, the volume of air within the pressure plethysmograph is large, compared to surface area of the subject and the walls of the container, so little heat exchange occurs. Hence the pressure-volume relationship within the plethysmograph is usually adiabatic (temperature changes with pressure). Whether the changes within the plethysmograph are isothermal, adiabatic or polytropic depends on the rapidity of the changes, the size of the subject in relation to the volume of the chamber, and the thermal conductivity of the materials in the walls of the plethysmograph; the latter being the least important of the factors listed, although low conductivity does favour adiabatic conditions. A lack of structural rigidity with the movement of the subject within the plethysmograph and/or the presence of compressible material within the chamber, particularly chairs with foam-padding and loose fluffy articles of clothing, can lead to unpredictable errors.

The smaller the volume of a plethysmograph with respect to the subject, the better the signal-to-noise ratio of the volume signal. On the other hand, small chambers may be cramped, uncomfortable and claustrophobic, and may result in an excessive build-up of heat, pressure and even carbon dioxide, as well as an increase in the likelihood of errors due to polytropic conditions. Too large a chamber means that the signal reaching the internal pressure transducer will be so small that the volume measured is inaccurate. The compromise is that plethysmographs for use in adults and older children should have a volume 100-300 times the volume being measured, i.e. a plethysmographic volume of 600$1,000 \mathrm{~L}$. This will ensure that the pressure changes in the plethysmograph will be small compared to alveolar 


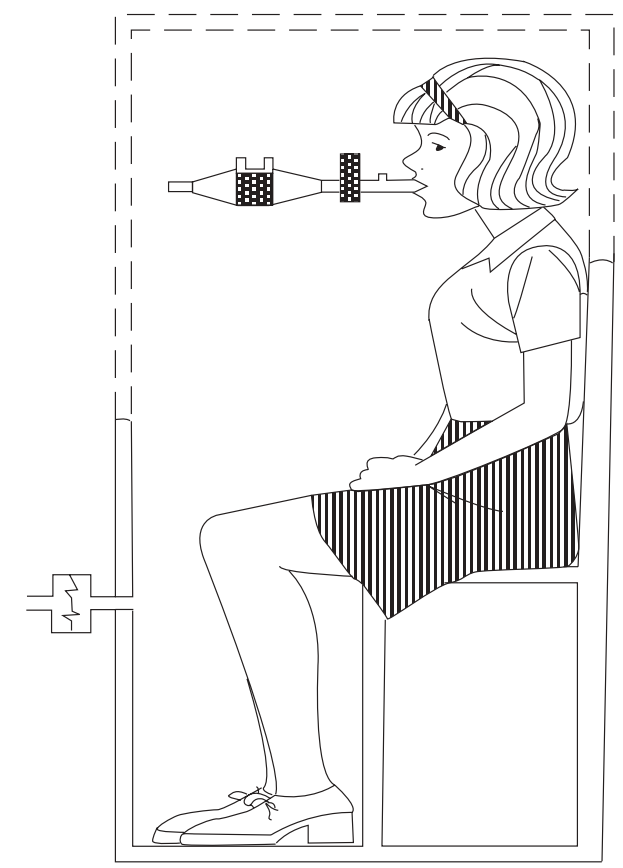

Fig. 1. - Schematic diagram of a variable pressure plethysmograph. In this example, the pneumotachograph is fully contained within the plethysmograph. A sensitive pressure transducer mounted in the walls measures the volume change (due to compression and rarefaction of the gas in the plethysmograph) in the subject. During the measurement of thoracic gas volume (TGV), the subject will be occluded by an electrically activated shutter valve at the airway opening. (Complements of L. Landau).

pressure changes and will not interfere with normal breathing, but large enough to be measured accurately. In infant plethysmographs, the requirement for quick and easy access to the infant usually results in a plethysmographic volume that is in the order of 50-100 $\mathrm{L}$ (500-1,000 times the lung volume being measured). While this places stringent demands on the sensitivity of the transducers being used, it facilitates adiabatic conditions.

The plethysmograph is usually open to the atmosphere by a small leak of controlled dimensions, for example, a narrow tube with an internal diameter of $0.05-2 \mathrm{~mm}$ and a length of $30-60 \mathrm{~cm}$, resulting in a mechanical time constant (compliance of the gas in the plethysmograph times the leak resistance) of 10-50 s, ideally in the order of $10 \mathrm{~s}$. This controlled leak minimizes slowly-occurring pressure changes, such as thermal drift, that are not related to respiratory manoeuvres. The shorter the time constant, the less the problems with thermal drift, but this may be at the cost of inaccuracies in the measurement of slowly-occurring events. It is important that the leak be constant. Inadvertent leaks, caused by poorly fitting doors or other open orifices, may lead to serious errors.

Because of the relatively large volume of the chamber compared to the small changes in volume of the subject, the transducer measuring changes in the chamber pressure must have an operating range in the order of $0.001-0.01 \mathrm{kPa}\left(0.01-0.1 \mathrm{cmH}_{2} \mathrm{O}\right)$. Thermal drift, particularly in infant plethysmographs, may give rise to pressure changes as much as $0.1 \mathrm{kPa}$, necessitating a larger working range of the transducer. Transducers of this sensitivity respond to the small changes in atmospheric pressure that accompany events such as opening or closing of a door in the room. One way of increasing the stability of the pressure signal is to reference the pressure changes within the interior of the plethysmograph to a reference chamber. The latter may be much smaller than the plethysmograph (i.e. in the order of $1: 100$ or less [21]). It is open to the room by a constant leak of controllable magnitude, so that the reference chamber has the same time constant as the plethysmograph [21].

Two time constants influence the behaviour of the plethysmograph, one due to the controlled leak, the mechanical time constant, and the other due to the volume of the chamber and thermal conductivity of the walls, the thermal time constant. Both influence the lower range of the frequency response characteristics. The thermal time constant may be measured by closing the controlled leak (providing this makes the plethysmograph airtight) and, with a syringe, suddenly injecting a sufficient quantity of air to increase the pressure inside the plethysmograph without saturating the pressure transducer. The pressure inside rises instantly and then falls exponentially towards a plateau (in the absence of leak). The ratio between the values of the initial rise and of the plateau is $\gamma$; the ratio of the specific heat of air at constant pressure and at constant volume [21] is 1.4 for air and other diatomic gases. The thermal time constant is the number of seconds required for the signal to fall to $63 \%$ of the value between initial and final equilibrium value [21]. If the controlled leak is opened and the procedure repeated, the overall time constant can be calculated. The overall response may be approximated by:

$$
\Delta P \text { pleth }=P \mathrm{~B} \times(\Delta V \text { pleth } / V \text { pleth }) \times\left[1+(\gamma-1) \times \mathrm{e}^{-\mathrm{t} / \tau \mathrm{th}}\right] \times \mathrm{e}^{-\mathrm{t} / \mathrm{\tau m}}
$$

where $\Delta P$ pleth is the change in pressure with time, $\Delta V$ pleth is the volume added with the syringe, $V$ pleth is the volume of the plethysmograph, $\tau$ th is the thermal time constant and $\tau \mathrm{m}$ is the mechanical time constant. With the mechanical leak closed, the $\tau \mathrm{m}$ is $\infty$ so the $\tau$ th can be calculated. With $\tau$ th known and the leak open, the $\tau \mathrm{m}$ can be calculated. The greater the time constant due to the controlled leak, the better the frequency response in the low range but the greater the problems with slowly-occurring nonrespiratory changes.

In practice, it is the total time constant that is important, and: when it is long compared with the panting or breathing cycle, changes in pressure within the chamber are completely adiabatic; when it is of the same order of magnitude, they may be polytropic; and when it is much shorter, they may be isothermal, as is the case within the lungs. Adiabatic conditions have a simple predictable relationship between pressure and volume within the plethysmograph; whereas, predicting polytropic conditions requires more complicated mathematical manipulation [21]. The proximity to isothermal or adiabatic conditions is dependent on the rapidity of the changes. The equation that relates changes in pressure and volume under adiabatic conditions is slightly more complicated than Boyle's Law because it includes the ratio of the specific heat of air at constant volume 
and constant pressure. However, if calibration is performed under adiabatic conditions, measurements are made under the same conditions, and changes in pressure and volume are relatively small, the relationship between $\Delta P$ pleth and $\Delta V$ pleth can be adequately approximated as a linear function.

Plethysmographs used in adults or older children, commonly have a thermal time constant in the order of 1 min combined with a leak time constant of 10-20 s. Using appropriate transducers, this will result in a flat frequency response from as low as $0.1-0.2 \mathrm{~Hz}$ to as high as $10-20 \mathrm{~Hz}$, adequate for most measurements. Specifically, if the combined time constant is at least $10 \mathrm{~s}$, the attenuation of the signal at a frequency of 0.1 $\mathrm{Hz}$ will be 0.98 and for $0.2 \mathrm{~Hz}, 0.995$, clearly adequate for virtually any respiratory manoeuvres. For a more complete description of the thermal process, the reader is referred to the monograph by BATES [22].

In order to measure all lung volumes as well as any difference between the volume at the time of occlusion and the normal end-expiratory volume, there must be either an external spirometer or a pneumotachograph (that may vent either inside or outside the chamber, depending on design).

\section{Volume displacement plethysmograph}

The modern volume displacement plethysmograph described by MEAD [19] is a rigid chamber, 300-600 L in volume (fig. 2). Part of the chamber opens directly into the base of a spirometer with low inertia, usually a Krogh type instrument connected to a rotational or linear displacement transducer. With the subject breathing from outside the chamber, the spirometer will measure large changes in lung volume, forced vital capacity (FRC) manoeuvres, etc. When the airway is occluded and the subject pants, small changes in volume due to thoracic gas compression are also accurately measured. With proper balancing (such as the use of springs to support the bellows of the spirometer and a low resistance pivoting device) the weight may be compensated and resistance of the system may be negligible, but the mass of the spirometer is significant, so inertia becomes a major determining factor in the frequency response. During rapid changes in volume, the spirometer is unable to follow the volume changes, which lead to compression of the air in the plethysmograph as the subject breathes in and rarefaction as the subject breathes out. During the transition between the two, the inertia of the bellows leads to oscillations or overshoot of the volume signal.

Placing felt-padding between the chamber and the spirometer dampens the signal, reducing overshoot and oscillations. The degree of damping should be tested by the introduction of a step function (rapid injection into the plethysmograph of a known quantity of air using a syringe). In an underdamped system (i.e no feltpadding) there will be an overshoot of the volume signal followed by oscillations of diminishing magnitude until a stable volume is reached. In an overdamped system (i.e. too much felt-padding) there will be a slow rise of the volume signal to its final value. The goal is critical damping, which is sufficient felt-padding to en-

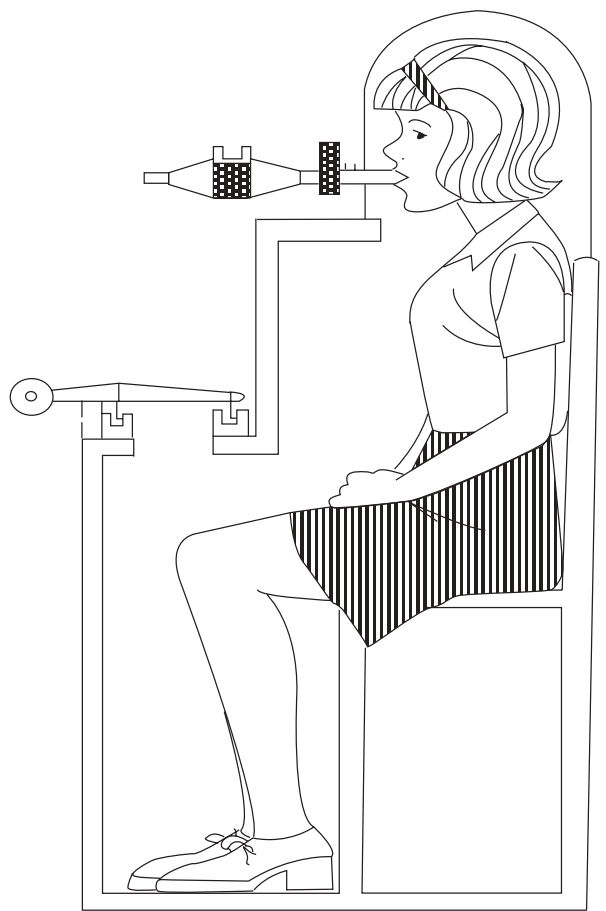

Fig. 2. - Schematic diagram of a volume displacement plethysmograph. The subject breathes to and from the plethysmograph through a pneumotachograph that exits outside. Volume changes are measured by a Krogh spirometer mounted within the wall of the plethysmograph. During the measurement of thoracic gas volume (TGV), the subject will be occluded by an electrically-activated shutter valve at the airway opening. The felt-damping below the spirometer and the pressure transducer for "pressure compensation" are not shown. (Complements of L. Landau).

sure that the volume signal rises rapidly, but without overshoot or oscillations (fig. 3). Once this is achieved, the plethysmograph can be "pressure compensated" by the addition of a proportion of the small variations of internal pressure that accompany the rapidly changing volumes because of the inertia of the spirometer. Since the magnitude of the internal plethysmographic pressure is proportional to the instantaneous "error" between the "true" volume and that measured by the spirometer, the addition of part of the internal pressure to the volume signal (pressure compensation) should improve the frequency response from the order of $4-5 \mathrm{~Hz}$ in the uncompensated plethysmograph to a value in the order of 8 Hz. This is still less than that of a variable pressure plethysmograph [21].

Within the chamber of the plethysmograph, the subject produces heat which gives rise to constant thermal drift, which is usually controlled by air-conditioning the chamber. Because the bellows of the spirometer has a finite range, it requires frequent adjustment. Difficulty in achieving control over thermal drift and the need for frequent adjustments of the bellows position has limited the extent to which these techniques can be automated, thereby reducing the popularity of the volume displacement plethysmograph as new, more automated products become available.

The major advantage of the volume displacement plethysmograph is its ability to measure large respiratory changes in volume (i.e. vital capacity manoeuvres) 
a)

b)

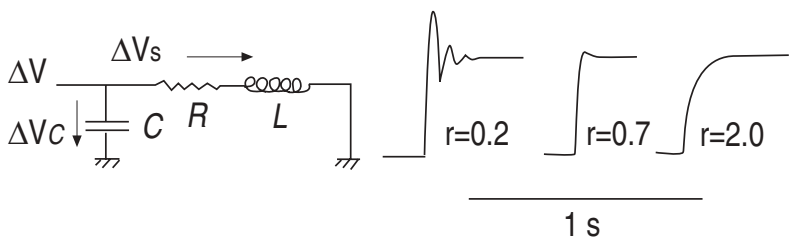

c)

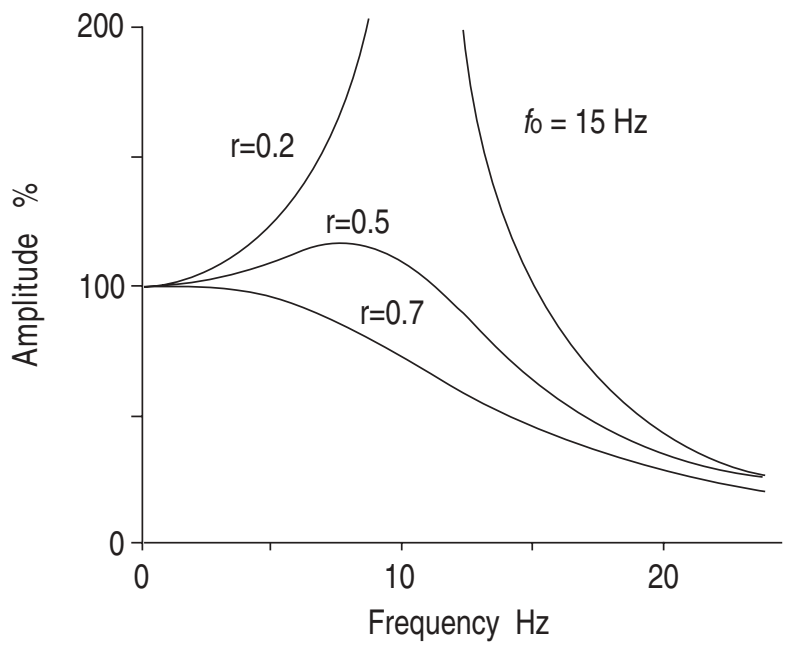

Fig. 3. - a) The electrical analogy of "critical damping". $C$ : capacitance; $R$ : resistance; $L$ : inertance; $\prod I T$ : earth; $\Delta V$ : difference in voltage either across the resistance and inertance $(S)$ or across the capacitor (C). b) The response to a step function: when the damping ratio is too low $(\mathrm{r}=0.2)$, there are oscillations around the final value; when it is too high $(\mathrm{r}=2.0)$, there is a slow response. Critically damped $(\mathrm{r}=0.7)$, the signal rises rapidly to its final value, with minimal or no overshoot. c) The amplitude response to a sinusoidal signal between 0 and $20 \mathrm{~Hz}$. In the underdamped situation, the system resonates, in this case at a resonant frequency $\left(f_{\mathrm{o}}\right)$ of $15 \mathrm{~Hz}$. (Reprinted with permission from [21]).

when the subject is breathing air from outside the chamber. Furthermore, during forced expiration, it measures both the volume that the subject expires and that compressed in the chest. The latter may be considerable in subjects with hyperinflation and airway obstruction [23].

\section{Flow plethysmographs}

In theory, the flow plethysmograph should be an ideal compromise between the variable pressure and volume displacement plethysmographs (fig. 4). Absolute rigidity of the walls is not necessary, problems with thermal time constants are minimized, and the frequency response, after pressure compensation, should be close to that of a variable pressure plethysmograph. Changes in volume of the lungs are measured by integrating the gas flow in and out of the chamber as measured by the differential pressure across either a capillary-type pneumotachograph or a wire mesh screen (25 $\mathrm{\mu m}$ mesh) mounted on the wall of the plethysmograph. The latter is almost a pure resistance; the former has both resistance and inertance. The sensitivity of the screen-type pneumotachograph to low flows can be increased by adding several layers of low resistance screen but this also increases resistance and, hence, the time constant, thereby reducing the frequency response. A $25 \mu \mathrm{m}$ mesh screen with a $14 \mathrm{~cm}$ diameter can be expected to have a linear range up to $15 \mathrm{~L} \cdot \mathrm{s}^{-1}$ and a resistance in the order of $0.003 \mathrm{kPa} \cdot \mathrm{L}^{-1} \cdot \mathrm{s}$ per layer of screen [21]. This allows maximal flow to be measured during forced expiration. For measurements of TGV and airway resistance, an $8 \mathrm{~cm}$ diameter screen with a resistance in the order of $0.01 \mathrm{kPa} \cdot \mathrm{L}^{-1} \cdot \mathrm{s}$ is suitable.

Pressure compensation increases the frequency response as in volume displacement plethysmographs [21]. For plethysmographs equipped with a capillary-type pneumotachograph, this requires a separate sensitive pressure transducer, whereas for plethysmographs equipped with wire mesh screen, the same transducer can be used to measure flow across the screen and pressure inside the plethysmograph. As in other types of plethysmographs, the use of a reference chamber with time constant characteristics similar to those of the plethysmograph can decrease the influence of ambient pressure variations. One of the problems with flow plethysmographs can be caused by a "zero drift" of the integrator, frequently caused by thermal drift or an "electrical zero" that does not correspond to true zero flow across the mesh. Drift of the volume signal can be ignored if the respiratory manoeuvre happens over a very short period of time (i.e. the panting manoeuvre). Alternatively, there are a variety of ways to compensate for thermal drift. One is to bias the integrator so that it "sees" only the gas flow caused by manoeuvres of the subject [21]. Another is to use an analogue or digital high pass filter, the equivalent of the "controlled leak" in the variable pressure plethysmograph. With proper pressure compensation, flow plethysmographs should have an adequate frequency response to $15-20 \mathrm{~Hz}$.

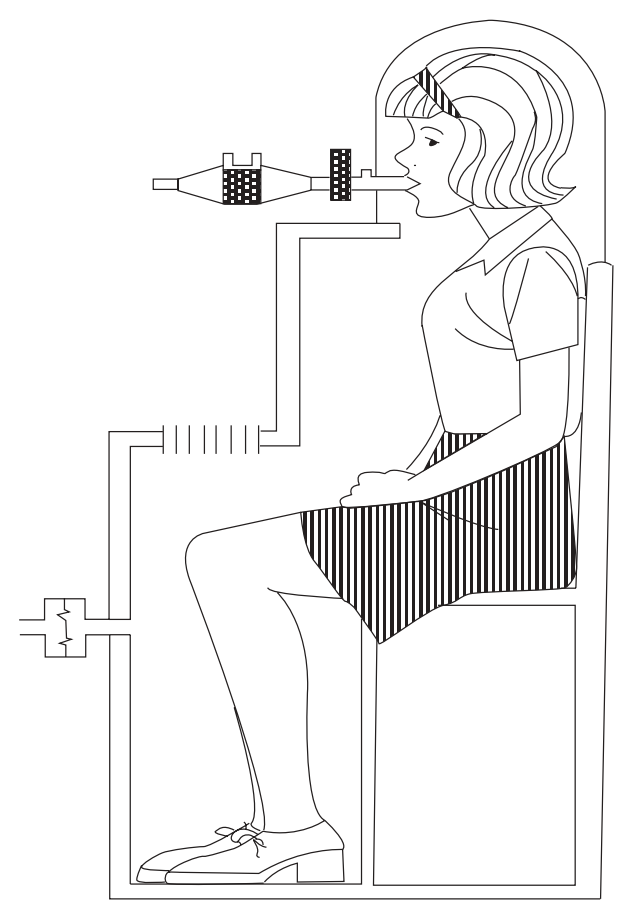

Fig. 4. - Schematic diagram of a flow plethysmograph. It differs from the volume displacement plethysmograph in that the spirometer has been replaced by a wire mesh flow sensor. In this case, the pressure transducer used for "pressure compensation" is shown in the wall. (Complements of L. Landau). 


\section{Variable pressure-flow plethysmographs}

The essential difference between variable pressure and flow plethysmographs is that the former are closed, whereas the latter are opened to the atmosphere through a pneumotachograph. A flow plethysmograph can be converted into a variable pressure plethysmograph simply by occluding the pneumotachograph orifice. Versatility is increased if a plethysmograph is built with an orifice which can either be closed (variable pressure plethysmograph) or opened to a pneumotachograph (flow plethysmograph), making it adaptable to the particular respiratory manoeuvre of interest. For example, the measurement of TGV could be performed using the variable pressure mode and a flow-volume curve could be performed using the flow mode. This would eliminate errors in flow-volume curves associated with the measurement of volume at the airway opening rather than the actual lung volume during the manoeuvre [23].

\section{Methodology}

The volume change of the intrapulmonary gas is usually achieved by panting against an occlusion at the airway opening [4]. The panting manoeuvre has been used for the determination both of FRCpleth and airway resistance (Raw). The original [4] justification for the shallow panting manoeuvre was threefold: to minimize temperature, saturation and respiratory quotient effects; to improve the signal to thermal drift ratio; and, finally, to minimize the contribution of the glottis to total $R$ aw, a factor which was later given further support [24]. Historically, changes in volume and pressure during panting were displayed on a calibrated oscilloscope and the slope of that tracing $(\Delta V / \Delta P)$ could be determined by rotating an overlaid template of parallel lines. With the advent of storage oscilloscopes and computers, the ability to measure $\Delta V / \Delta P$ has become easier, since an instantaneous relationship can be retained on the screen. This allows manoeuvres other than panting [8].

Many young children have difficulty with the standard panting manoeuvre but can generate adequate rarefaction of intrathoracic gas during an inspiratory effort against an obstruction at end-expiration [8]. Although the inspiratory technique was originally mentioned by DuBors et al. [4], it did not initially gain favour, presumably in part because of technical difficulty in measuring the slope traced by a rapidly moving single dot on an oscilloscope screen. With modern data acquisition systems, children who have difficulty panting can be encouraged to make a single inspiratory effort at the time of an end-expiratory occlusion [8], although, as mentioned previously, the use of the complete version of Boyle's Law, Equation (6), significantly improves accuracy with this method [9].

Two potential disadvantages of this technique are that any leaks, which usually present as a loop in the $\Delta V /$ $\Delta P$ tracing during the panting manoeuvre, are hard to detect during a single inspiratory effort, and excessive thermal drift, which presents as a difference in slope between the inspiratory and expiratory phase of a pant, may not be appreciated without the expiratory phase. Hence, the manoeuvre must result in a rapid change in pressure and volume to avoid problems due to thermal drift. There are data to suggest that if duration of the inspiratory manoeuvre is in the order of $0.33 \mathrm{~s}$ thermal drift is not a factor and may play an insignificant role as long as it is under $0.8 \mathrm{~s}$ [9]. However, the use of a much slower inspiratory manoeuvre, such as would occur in an adult during quiet breathing when occluded at end-expiration, cannot be recommended at present since, there has so far been no rigorous evaluation of the accuracy of this technique, and the duration of the manoeuvre invites errors due both to thermal drift and polytropic conditions within the plethysmograph.

Accurate results can be obtained in infants who usually make repeated respiratory efforts against the occlusion at around $0.5 \mathrm{~Hz}$. As long as there is a tracing over time over all phases of the repeated respiratory cycle, corrections can be made for thermal drift [25].

\section{Calibration}

Regardless of the type of plethysmograph, a transducer capable of measuring $P$ ao up to at least $\pm 5 \mathrm{kPa}$, with a flat frequency response in excess of $8 \mathrm{~Hz}$ is essential [21]. The spirometer or pneumotachograph used for measuring tidal and forced inspiratory and expiratory volumes should meet published standards for precision of spirometric devices [26]. Volume calibration should be checked daily using a calibrating syringe, with a displacement that is in the same order of magnitude as the vital capacity of the subject. The volume signal should be adjusted to account for the difference between conditions in the syringe and BTPS, which will depend on the direction of the flow (i.e. inhaling will expose the pneumotachograph to ambient air, while exhalation exposes it to BTPS conditions). If the calibration is significantly different during inspiration and expiration, there will be a drift in the volume signal. The airway opening pressure transducer should be physically calibrated daily. The linearity both of the airway pressure transducer and volume output of the plethysmograph should be checked over the entire working range at least every 6 months. The linearity of the volume signal can be checked by injecting known small amounts of air with a syringe, (i.e. 5, 10, 20, 30 and 50 $\mathrm{mL}$ ).

The plethysmograph should also be calibrated daily using a calibrating volume signal that is of a magnitude and frequency similar to the panting manoeuvre, usually by a small reciprocal pump that delivers a sinusoidal volume signal of $20-50 \mathrm{~mL}$ for adult plethysmographs and 5-10 $\mathrm{mL}$ for infant plethysmographs. The pressure transducer is then adjusted to give a known signal for a known volume. The calibration signal (as well as the working range of the transducer) should be of the same order of magnitude as the volume change in the subject to prevent having to alter the amplification of the signal following calibration. Such alterations can introduce potential sources of error, such as saturation of the transducer if the calibration signal is much smaller than the physiological signal, or decreased signal-tonoise ratio, if the calibration signal is much greater. If the calibration is performed with the subject in the plethysmograph holding his breath, no further adjustment 
in the calibration is needed. If the plethysmograph is calibrated empty, the data from the pressure transducer, in other words, $\Delta P$ pleth, need to be reduced appropriately:

\section{(plethysmographic volume - subject volume)/ plethysmographic volume}

In practice, the subject's volume in litres is estimated from the weight in kilograms divided by 1.07 [27]. Errors in the weight of the subject will introduce small errors into the final measurement. Since babies do not hold their breath on request, the calibration can be carried out either by replacing the baby with a liquid filled bag of the same volume, or by adjusting the signal mathematically [25].

An absolute check on accuracy can be made by using a 3-4 L rigid container (e.g. an Erlenmeyer flask), that is filled with copper wool (to keep conditions isothermal within the flask and, thereby, analogous to the lung) and which can be connected both to the mouthpiece and to a rubber bulb [27] (fig. 5). The bulb can be squeezed at a frequency of $1 \mathrm{~Hz}$ by a subject (holding his breath) inside the plethysmograph. This rarefies and compresses the gas inside the plethysmograph allowing a "TGV" measurement. Since the volume of the container can be measured, and the volume of the copper wool (calculated from weight and density) subtracted, the value of the "TGV" can be checked. In this case, water vapour pressure should not be subtracted from barometric pressure when calculating TGV. Alternatively, the resulting TGV can be adjusted by multiplying it by $\left(P \mathrm{~B} /\left(P \mathrm{~B}-\mathrm{PH}_{2} \mathrm{O}\right)\right.$. The accuracy of the plethysmograph in measuring "TGV" of the container should be $\pm 50 \mathrm{~mL}$ or $3 \%$, whichever is greater based on a mean of five determinations. Similar checks can be made for infant plethysmography by using an appropriate physical analogue attached to a sinusoidal pump [25].

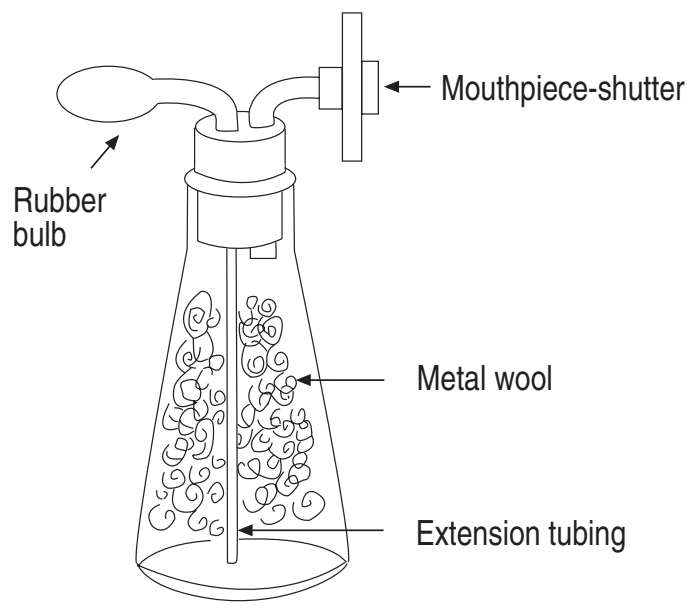

Fig. 5. - An Erlenmeyer flask of known volume that can be used to check the measurement of thoracic gas volume (TGV). Copper wool is placed in the flask to act as a heat sink, so that when the contents are compressed by squeezing the bulb, the changes in pressure and volume are isothermal. The volume of the copper wool can be obtained from weight and density and subtracted from the volume of the flask. (Reprinted with permission from [27]).

\section{Thermal drift}

Thermal drift due to temperature changes in the interior of the plethysmograph is common in all types. Hence, the plot of $\Delta V / \Delta P$ during a panting manoeuvre shows a systematic difference in slope between compression and rarefaction. If the subject has been breathing to atmosphere prior to the occlusion, the drift of the plethysmographic volume signal is usually upwards during the occlusion, as the subject warms the plethysmograph. If thermal equilibrium has been reached while the subject has been breathing to and from the chamber, there will be a reduction in the heat produced during the occlusion and the drift will be downward.

Providing the drift is not too large and is relatively constant with respect to time, there are mathematical procedures that can eliminate it. The simplest is to compute drift, the change in volume over time, and then adjust the plethysmographic volume signal proportionally during the occluded manoeuvre. During inspiratory and expiratory efforts against the occlusion, thermal drift influences the slope of $\Delta V / \Delta P$ in opposite directions, the difference in the two slopes being the thermal drift. There is an iterative method [28], where the regression coefficient (r) between $P$ ao and $V$ pleth is calculated while applying a correction factor proportional to time $(t)$ to the plethysmographic volume signal:

$$
V \text { pleth }=V \text { pleth }+\mathrm{K} \times t
$$

$\mathrm{K}$ may be positive or negative depending on direction of the drift. As $t$ is increased in small steps, the point where $\mathrm{r}$ is maximal is the correct product of $\mathrm{K}$ and $t$. No matter which method is chosen, the result corrects only for drift and will not change the relationship between $V$ pleth and $P$ ao due to gas compression or other reasons.

\section{Frequency response}

The ability of the signal output by the transducer to follow the applied signal both in time and magnitude constitutes the frequency response. The frequency response of commercial equipment should be stated by the manufacturer, and the user should be given detailed instructions of how to verify it. Ideally, the frequency response should be measured at least once every 6 months and after any significant change in the apparatus, for example, repairs or replacement of a transducer. This is most commonly accomplished with the application of a sinusoidal volume signal where the frequency can be varied [21]. It is usually expressed as a plot of phase shift in degrees and attenuation of the plethysmograph volume compared to the applied volume versus the logarithm of the frequency (Bode plot) (fig. 6). This may be used both with attenuation and frequency logarithmic [29] or, as adapted for respiratory measurement, with the attenuation axis linear [30]. For a first order system, such as a non-pressure-compensated flow box with a wire mesh screen, the attenuation at any given frequency in response to a sinusoidal forcing function [29], with frequency expressed in radians per second $(\omega)$, will be $1 /\left(1+\omega^{2} \tau^{2}\right)^{1 / 2}$. 
Another technique for calculating frequency response is to evaluate the response to a step function (of a constant magnitude $\mathrm{K}$ ). This can be done by rapidly introducing a small volume of air into the plethysmograph with a syringe. In a flow or properly tuned volume displacement plethysmograph, the volume signal will increase exponentially and reach a plateau. The rapidity of the rise (assuming the time to introduce the air is negligible) is a test of the high frequency response characteristics of the plethysmograph. The time constant of the rise $(\tau)$ can be calculated from the point where the equation $\mathrm{K} \times\left(1-\mathrm{e}^{-\mathrm{t} / \tau}\right)$ reaches $63 \%$ of its final value. If the time to introduce the air into the system is not negligible, the calculated high frequency response will represent both the response of the plethysmograph and the rapidity of the introduction of the air. It is generally recommended that the minimum adequate frequency response be five times the frequency of the signal being measured [21]. For a pant at $1 \mathrm{~Hz}$, this means fidelity of the signal at $5 \mathrm{~Hz}$ but, for an inspiratory effort, the requirement could be greater, depending on the rapidity of the effort. To ensure that panting frequencies slightly above $1 \mathrm{~Hz}$ will not lead to problems, the minimum acceptable frequency response should result in accuracy at $8 \mathrm{~Hz}$, generally felt to be the best frequency response for a pressure-compensated volume displacement plethysmograph. Constant volume variable pressure plethysmographs and flow plethysmographs should be able to perform better than $8 \mathrm{~Hz}$, usually with a flat frequency response between 10 and $20 \mathrm{~Hz}$.
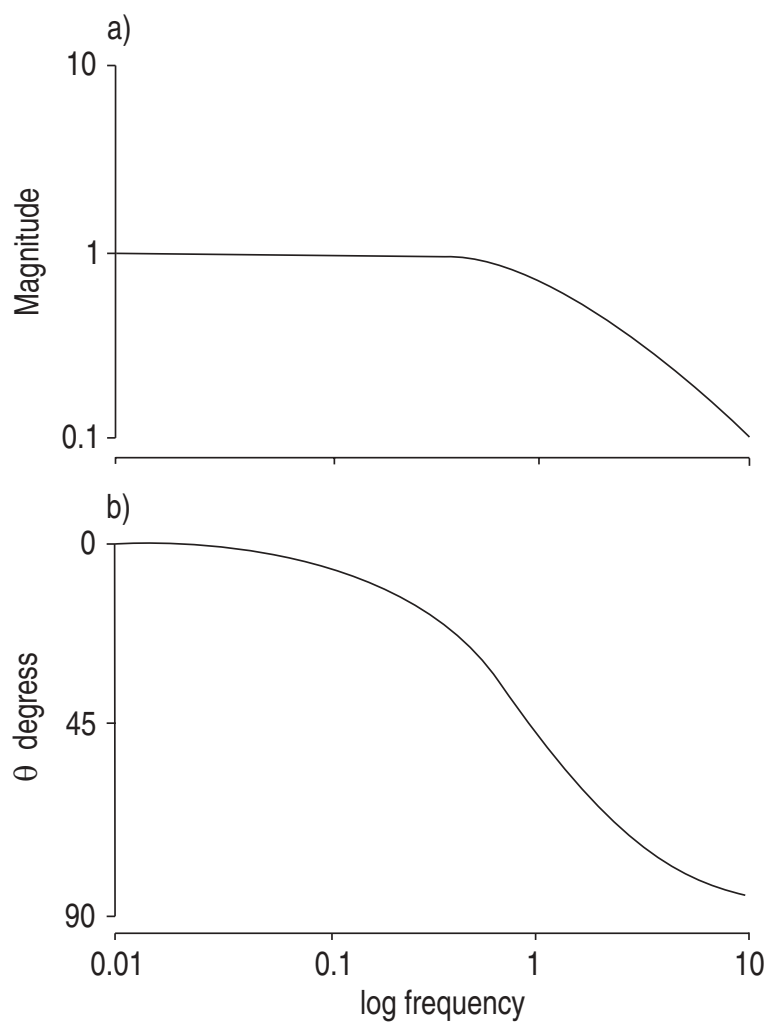

Fig. 6. - Illustration of a Bode plot, where: a) the gain (Magnitude) of the system; and $b$ ) the phase shift $(\theta)$ in degrees is plotted against the logarithm of the applied frequency. (Reprinted with permission from [21]).

\section{Measurement techniques}

The equipment should be adjusted so that the subject can sit comfortably in the chamber and reach the mouthpiece without having to flex or extend the neck. For children, this may require special adjustments. After suitable explanation of the technique, the subject then enters the plethysmograph and breathes through the mouthpiece with a noseclip in place. The door is closed and an initiation period is given to allow thermal transients to stabilize and the subject to relax during tidal breathing, so that a baseline representing the "relaxed" FRC can be determined. Breathing to and from the outside reduces the heating of the chamber and eliminates the possibility of the build-up of $\mathrm{CO}_{2}$. However, it may increase the apparatus dead space (specifically the volume between the mouthpiece and the shutter valve) or damage the highly sensitive pressure transducer in the variable pressure plethysmograph, as a result of the large volume changes that ensue under these conditions if the chamber remains closed. Alternatively, breathing within the chamber results in rapid heat production that may speed up the equilibration process (although the temperature at the point of stability may be higher).

The chamber temperature increases rapidly after closing the door and then rises towards body temperature. Due to heat exchange through the walls, the equilibrium temperature is always below body temperature (unless ambient temperature is $37^{\circ} \mathrm{C}$ ) and it depends very much on the metabolic activity of the subject. Testing may commence once the initial rapid temperature rise has occurred and the continuing thermal drift in internal pressure is less pronounced. Testing initiated prior to thermal stability is prone to errors and should be avoided. Thermal stability is indicated when the change in internal pressure over time is both small and constant with respect to time.

Prior to any manoeuvre, the variable pressure plethysmograph is vented to atmosphere using a large bore valve. This eliminates the positive pressure due to warming of the interior gas, which is too great to be vented by the controlled leak. If this initiation period is prolonged, the plethysmograph should be vented frequently, so that the pressure build-up will not overload the internal pressure transducer. In a volume displacement plethysmograph, the spirometer is brought to the midline position prior to any respiratory manoeuvre.

The subject is instructed about the manoeuvre. He is asked to support his cheeks and chin firmly with both hands. Any dentures should remain in place, since their removal would increase the floppiness of the cheeks. Breathing is continually monitored by the operator, in order to establish a stable baseline representing FRC. The airway is usually occluded by closure of a shutter valve when the subject is at FRC. The subject makes a series of gentle panting manoeuvres $( \pm 1 \mathrm{kPa})$ at a frequency of about $1 \mathrm{~Hz}$. Panting at this low frequency has been shown to greatly reduce or eliminate errors due to flow resistive losses caused by the upper airway acting as a capacitive shunt [6], yet is sufficiently fast to avoid problems with polytropic conditions within the interior of the plethysmograph. It is important that the volume of gas between the subject and the shutter valve be kept to a minimum. While the conditions in 
the lung are essentially isothermal, those in the apparatus dead space can be adiabatic or polytropic, making it extremely difficult to correct for dead space accurately.

During the panting manoeuvre, the operator monitors the $\mathrm{X}-\mathrm{Y}$ plot of the $\Delta P-\Delta V$ relationship. The panting should result in a series of almost superimposable straight lines separated only by small thermal drift. Deviations from this, such as looping or nonlinear segments, can occur with glottic closure, leaks or inadequate cheek support. In order to allow for the detection of "looping", the electrical gain of both the $\Delta P$ and $\Delta V$ should be such that the slope of the $\Delta V / \Delta P$ is $30-60^{\circ}$.

A series of 3-5 panting manoeuvres that have a satisfactory $\Delta \mathrm{P}-\Delta V$ phase relationship are carried out, after which, the shutter is opened and the subject is instructed to expire maximally followed by a slow inspiratory vital capacity (VC) manoeuvre up to total lung capacity (TLC). However, subjects with severe pulmonary disease and/or dyspnoea may have difficulty with an expiratory manoeuvre following what is essentially $3-5 \mathrm{~s}$ of apnoea during the panting manoeuvre. As an alternative, such subjects can be instructed either to take two or three tidal breaths after the panting manoeuvre followed by a maximal expiration, or to inspire maximally to TLC and then perform a slow maximal expiration. For patients with lung disease, the residual volume (RV) may differ, depending on whether the panting manoeuvre is followed by an inspiratory or an expiratory manoeuvre $[31,32]$. When occluding at volumes other than FRC, each TGV is adjusted to give the FRCpleth.

When using a variable pressure plethysmograph, an external spirometer or a pneumotachograph is used to measure maximal inspiratory and expiratory volumes, with the plethysmograph vented to atmosphere to avoid overloading the plethysmograph pressure transducer. In the volume displacement or flow plethysmograph, the subject breathes from outside the chamber and the plethysmograph measures the total change in volume of the subject. This vital capacity includes both the volume of expired gas and any volume of compression [23] resulting from positive pressure in the alveolar space. While the volume change due to compression will be small in a normal subject, it may be considerable in subjects with marked airway closure at low lung volumes, who generate large positive pleural pressures [23]. Hence, the VC measured in a flow or volume plethysmograph may be significantly greater than that measured at the airway opening. The effect of these different measurement techniques should be understood when expressing the results.

In a flow or volume displacement plethysmograph, where the same device is used to measure all volumes, it must be noted that the volume signal during the VC manoeuvre is orders of magnitude greater than that obtained during panting, requiring an adjustment of the electrical gain of the system.

There are a number of ways to calculate lung volumes from FRCpleth, and the difference between the various options are relatively small [33]. A reasonable recommendation [33] is that TLC be the average of the acceptable values of FRCpleth and the largest inspiratory capacity (IC). The expiratory reserve volume (ERV) is derived from the maximal expiration, and IC + ERV represent $\mathrm{VC}$, with $\mathrm{RV}$ being the difference between TLC and VC.

For children old enough to co-operate fully with the procedure, there should be little difference in plethysmographic techniques compared to adults. The age of children capable of "full co-operation" is clearly influenced by the paediatric experience of the individual performing the testing, but, in an appropriate setting, most children 8 years old or more can perform the panting manoeuvre adequately.

\section{Quality control}

There should be at least two recognized pants displayed on an X-Y plot (preferably with the option of a time-based plot as well) at a frequency of $0.5-1 \mathrm{~Hz}$ and where $\Delta P$ ao does not exceed $2 \mathrm{kPa}$. With only one manoeuvre, it is extremely difficult to ascertain the existence and magnitude of thermal drift, whereas superimposed multiple manoeuvres clearly imply a degree of reproducibility. Following correction for thermal drift, calculations of goodness-of-fit using a least-squares linear regression of $P$ ao on $V$ pleth is desirable. Low correlation coefficients (r) may result from improper technique, e.g. glottic closure during the manoeuvre, leaks in the system, excessive and variable thermal drift or other problems. The X-Y plot may show looping: if the upper airway acts as a shunt capacitance in patients with severe obstructive disease when panting at a high frequency $[10,11]$; if there is a poor frequency response of the equipment; or because of inhomogeneity of alveolar pressure swings. In automated systems, the value of $r$ should, ideally, be provided to the operator with the suggestion that values less than 0.9 , and ideally 0.95 , should raise operator concern and result in some trouble-shooting. Other causes of a low $r$ include a poor signal-to-noise ratio, due, for example, to instability of local environmental conditions which may be overcome by efforts to achieve barometric stability. In the end, the operator must decide whether to accept or reject the data based on his decision that this does or does not represent a valid measurement. Single inspiratory manoeuvres should yield virtually superimposable X-Y plots, and values of FRCpleth within $5 \%$ of each other.

At least monthly, preferably weekly, two reference subjects should have FRCpleth, RV and TLC measured. Values that differ significantly ( $>5 \%$ for FRC and TLC or $>10 \%$ for RV) from the previous established means for measurements on the same subject require immediate investigation.

\section{Plethysmography in infants}

Detailed descriptions of plethysmographic measurements of FRCpleth in infants have been published [12, $25,34-36]$. Whilst considerable effort has gone into trying to standardize the measurement of lung volumes [25], there are difficulties in making suitable recommendations for infants too young to be able to co-operate in any way with the testing procedure. Relatively few centres make these measurements, and there is a lack of standardized equipment. Most infant equipment 
is "home-made" and all the necessary specifications for comparative conclusions are rarely presented in the literature. As with equipment for adults, volume and frequency characteristics of the infant plethysmograph must be known and shown to be adequate (i.e. at least five times the frequency of the respiratory manoeuvres being measured). Generally, most infant plethysmographs are the variable pressure type, although a flow plethysmograph suitable for measuring FRC has been reported [34]. Although the theory and principles of plethysmography in adults and infants are essentially the same, major differences exist in practice [25]. The major physiological difference between measurements in co-operative subjects and those in infants are related to sleep state and posture.

In the first month of life, measurements can be made during natural sleep, but in older infants sedation is usually required. Although in adults TGV is conventionally calculated from the slope of box pressure (or displacement volume) versus $P$ ao $(\Delta V / \Delta P)$, the additional use of time-based traces is strongly recommended for calculations in infants. The latter approach makes it possible: to assess variations in end-expiratory levels more accurately; to correct for thermal or metabolic drifts during airway occlusion; and to occlude the airway at any phase of the tidal breath (e.g. at end-inspiration) and, subsequently, correct to the end-expiratory level. During the occluded respiratory efforts, $\Delta P$ pleth should be in phase with and the same shape as $\Delta P$ ao. $A$ reduction in upper airway tone such that $\triangle P$ A does not equal $\Delta P$ ao during the occlusion may be reflected as a phase lag between $\Delta P$ pleth and $\Delta P$ ao.

Infants do not pant but make relatively low frequency respiratory efforts (around $0.5 \mathrm{~Hz}$ ) against the occlusion, which increases the problems of thermal drift when compared to adults panting at $1 \mathrm{~Hz}$. With a variable pressure plethysmograph, the combined thermal and mechanical time constant should be at least $10 \mathrm{~s}$, as in plethysmographs used in adults. This will provide an adequate frequency response down to $0.1-0.2 \mathrm{~Hz}$. If the box is calibrated at the approximate frequency of the respiratory efforts against the occlusion, any errors introduced by polytropic conditions will be cancelled out in calibration [37]. Unless efforts are specifically made to reduce the thermal time constant (metallic walls, fan, etc.), conditions in infant plethysmographs are adiabatic over the range of frequencies encountered (i.e. 0.3-2 Hz [25]). This can easily be verified by the operator calibrating the system over the desired frequency range and ensuring that the recorded signal remains constant. If not, most potential problems can be corrected with appropriate software [38].

\section{Measurement techniques}

The sleeping infant is placed inside the plethysmograph and a face-mask attached to a pneumotachograph and shutter is sealed around the nose and mouth. The seal can be tested by recording at least five tidal breaths before occlusion to establish a stable end-expiratory level, and then briefly closing the shutter at end-inspiration. If the seal is tight, flow will be zero throughout the occlusion and the volume recorded will return to the expiratory baseline after the release of the shutter. Any increase in the volume baseline after release of the occlusion or decay of $P$ ao signal during occlusion suggests a leak [25, 39]. Ideally, the mask should be held in place with strapping to support the cheeks and, hence, reduce risk of shunting to the upper airways during the occlusion, but this is rarely done. After eliminating leaks, the plethysmograph is closed.

If a pressure plethysmograph is used, it is allowed to reach thermal equilibrium (as indicated by minimal drift of the box pressure signal). At least five tidal breaths (more, if end-expiratory level is unstable) should then be recorded before the airway is occluded. FRCpleth is conventionally measured by closing a shutter at end-expiration and allowing the infant to make 2-4 respiratory efforts against the occlusion, from which the relationship of $\Delta P$ pleth to $\Delta P$ ao is established. In practice, occlusion is frequently performed at end-inspiration, with subsequent subtraction of the volume above end-expiratory level at the moment of occlusion. This improves the signal-to-noise ratio, is better tolerated by most infants and reduces the incidence of glottic closure. The issue of which volume to occlude at is still under debate [1. 13]. In healthy infants, measurements made at end-inspiration and end-expiration agree within 5\% after correcting for the inspired tidal volume, thereby providing a simple and effective in vivo method of validating the accuracy of the measurements [40].

During the occlusion, the changes in box volume and $P$ ao should be strictly in phase. A loop appearing on an $\mathrm{X}-\mathrm{Y}$ display usually indicates a leak in the system or glottic closure. Three to five separate occlusions should be made in each infant, all obtained during quiet sleep [41].

Although no standardized approach to reporting FRCpleth values in infants has been established, it seems reasonable to report a mean value of at least three, preferably five, separate, technically satisfactory measurements. In healthy infants, FRCpleth measurements tend to be highly reproducible, with a coefficient of variation of less than $5 \%$ [25]. The variability may be greater in infants with respiratory disease [42], those who are not in quiet sleep [43], or those without a stable end-expiratory level before occlusion.

\section{Conclusions}

Since the original description of the clinical use of plethysmography by DuBoIs et al. [4], the field of plethysmography has undergone considerable change. Most of this has led to increased understanding of the physiology as well as technical advances with the equipment. As a result, measurements of functional residual capacity derived from plethysmography are now based on a better scientific foundation, with user friendly equipment within the fiscal and technical reach of most clinical respiratory laboratories.

\section{References}

1. Lanteri CJ, Raven JM, Sly PD. Should TGV be measured from end-inspiratory occlusions rather than 
end-expiratory occlusions in wheezy infants? Pediatr Pulmonol 1990; 9: 214-221.

2. Brown R, Hoppin FGJ, Ingram RHJ, Saunders NA, McFadden ERJ. Influence of abdominal gas on the Boyle's law determination of thoracic gas volume. $J$ Appl Physiol: Respirat Environ Exercise Physiol 1978; 44: 469-473.

3. Stocks J, Quanjer PhH. Reference values for residual volume, functional residual capacity and total lung capacity. Eur Respir J 1995; 8: 492-506.

4. DuBois AB, Botelho SY, Bedell GN, Marshall R, Comroe JH Jr. A rapid plethysmographic method for measuring thoracic gas volume: a comparison with a nitrogen wash-out method for measuring functional residual capacity in normal subjects. J Clin Invest 1955; 35: 322326.

5. Bohadana AB, Peslin R, Hannhart B, Teculescu DB. Influence of panting frequency on plethysmographic measurements of thoracic gas volume. J Appl Physiol: Respirat Environ Exercise Physiol 1982; 52: 739747.

6. Shore SA, Huk O, Mannix S, Martin JG. Effect of panting frequency on the plethysmographic determination of thoracic gas volume in chronic obstructive pulmonary disease. Am Rev Respir Dis 1983; 128: 54-59.

7. Brown R, Slutsky AS. Frequency dependence of plethysmographic measurement of thoracic gas volume. $J$ Appl Physiol: Respirat Environ Exercise Physiol 1984; 57: $1865-1871$.

8. Desmond KJ, Demizio DL, Allen PD, Beaudry PH, Coates AL. An alternate method for the determination of functional residual capacity in a plethysmograph. Am Rev Respir Dis 1988; 137: 273-276.

9. Coates AL, Desmond KJ, Demizio DL. The simplified version of Boyle's Law leads to errors in the measurement of thoracic gas volume. Am J Respir Crit Care Med 1995; 152: 942-946.

10. Stanescu DC, Rodenstein DO, Cauberghs M, Van de Woestijne KP. Failure of body plethysmography in bronchial asthma. J Appl Physiol: Respirat Environ Exercise Physiol 1982; 52: 939-948.

11. Rodenstein DO, Stanescu DC, Francis C. Demonstration of failure of body plethysmography in airway obstruction. J Appl Physiol: Respirat Environ Exercise Physiol 1982; 52: 949-954.

12. Godfrey S, Beardsmore CS, Maayan C, Bar-Yishay E. Can thoracic gas volume be measured in infants with airways obstruction? Am Rev Respir Dis 1986; 133: 245-251.

13. Helms P. Problems with plethysmographic estimation of lung volume in infants and young children. $J$ Appl Physiol: Respirat Environ Exercise Physiol 1982; 53: 698-702.

14. Beardsmore CS, Stocks J, Silverman M. Problems in measurement of thoracic gas volume in infancy. $J$ Appl Physiol: Respirat Environ Exercise Physiol 1982; 52: 995-999.

15. Rodenstein DO, Francis C, Stanescu DC. Airway closure in humans does not result in overestimation of plethysmographic lung volume. J Appl Physiol: Respirat Environ Exercise Physiol 1983; 55: 1784-1789.

16. LeSouef PN, Lopes JM, England SJ, Bryan MH, Bryan AC. Influence of chest wall distortion on esophageal pressure. J Appl Physiol: Respirat Environ Exercise Physiol 1983; 55: 353-358.

17. Coates AL, Davis GM, Vallinis P, Outerbridge EW. Liquid-filled esophageal catheter for measuring pleural pressure in preterm neonates. $J$ Appl Physiol 1989; 67: 889-893.

18. Castile RG, Brown R. More problems with Boyle's Law, or, "Vtg or not $V_{\mathrm{tg}}$, that is the question". Am Rev Respir Dis 1986; 133: 184-185.

19. Mead J. Volume displacement body plethysmograph for respiratory measurements in human subjects. $J$ Appl Physiol 1960; 15: 736-740.

20. Nolte D. Experimental studies on the existence of isothermal conditions in the human lung (International Symposium on Body Plethysmography, Nijmegen, 1968). Prog Respir Res 1969; 4: 102-108.

21. Peslin R. Body plethysmography. In: Techniques in the Life Sciences: Respiratory Physiology. 414th edn. Elsevier Scientific Publishers, County Clare Ireland. 1984; pp. $1-26$.

22. Bates JH. Correcting for the thermodynamic characteristics of a body plethysmograph. Ann Biomed Eng 1989; 17: 647-655.

23. Coates AL, Desmond KJ, Demizio D, Allen PD, Beaudry PH. Sources of error in flow-volume curves: effect of expired volume measured at the mouth $v s$ that measured in a body plethysmograph. Chest 1988; 94: 976-982.

24. Stanescu DC, Clement J, Pattijn J, Woestijne KP. Glottis opening and airway resistance. J Lab Physiol 1972; 32: 460-466.

25. Stocks J, Marchal F, Kraemer R, Gutkowski P, BarYishay E, Godfrey S. Plethysmographic assessment of functional residual capacity and airway resistance. In: Stocks J, Sly PD, Tepper RS, Morgan WJ, eds. Infant Respiratory Function Testing. New York, Wiley-Liss, 1996; pp. 191-239.

26. American Thoracic Society. Standardization of spirometry: 1994 update. Am J Respir Crit Care Med 1995; 152: $1107-1136$.

27. Zarins LP, Clausen JL. Body Plethysmography. In: Clausen JL, ed. Pulmonary Function Testing Guidelines and Controversies: Equipment, Methods, and Normal Values. New York, Academic Press, 1982; pp. 141-153.

28. Peslin R, Gallina C, Rotger M. Methodological factors in the variability of lung volume and specific airway resistance measured by body plethysmography. Bull Eur Physiopathol Respir 1987; 23: 323-327.

29. Jackson AC. Dynamic response of transducers used in respiratory physiology. In: Techniques in Life Sciences, Respiration Physiology. Elsevier Scientific Publishers, Ireland, 1992; pp. 1-18.

30. Vallinis P, Davis GM, Coates AL. A very low dead space pneumotachograph for ventilatory measurements in newborns. J Appl Physiol 1990; 69: 1542-1545.

31. Christie RV. The elastic properties of the emphysematous lung and their clinical significance. J Clin Invest 1934; 13: 295-302.

32. Dayman H. Mechanics of airflow in health and emphysema. J Clin Invest 1951; 30: 1175-1185.

33. Bohadana AB, Teculescu DB, Peslin R, Jansen da Silva JM, Pino J. Comparison of four methods for calculating the total lung capacity measured by body plethysmography. Bull Physiopathol Respir (Nancy) 1980; 16: 769-776.

34. Marchal F, Duvivier C, Peslin R, Haouzi P, Crance JP. Thoracic gas volume at functional residual capacity measured with an integrated-flow plethysmograph in infants and young children. Eur Respir J 1991; 4: 180-187.

35. Stocks J, Godfrey S. Specific airway conductance in relation to postconceptional age during infancy. J Appl 
Physiol: Respirat Environ Exercise Physiol 1977; 43: 144-154.

36. Stocks J, Levy NM, Godfrey S. A new apparatus for the accurate measurement of airway resistance in infancy. J Appl Physiol: Respirat Environ Exercise Physiol 1977; 43: 155-159.

37. Stocks J, Fletcher ME. On the effect of the thermodynamics of an infant plethysmograph on the measurement of thoracic gas volume (Letter and Comment). Pediatr Pulmonol 1991; 10: 63-64.

38. Sly PD, Lanteri CJ, Bates JH. Effect of the thermodynamics of an infant plethysmograph on the measurement of thoracic gas volume. Pediatr Pulmonol 1990; 8: 203-208

39. Stocks J, Nothen U, Sutherland P, Hatch DJ, Helms P. Improved accuracy of the occlusion technique for asses- sing total respiratory compliance in infants. Pediatr Pulmonol 1987; 3: 71-77.

40. Dezateux CA, Fletcher ME, Stocks J. Plethysmographic measurements of thoracic gas volume in infants: timing of occlusions and repeatability. Eur Respir J 1992; 5: $36 \mathrm{~S}$.

41. Prechtl HF. The behavioural states of the newborn infant (a review). Brain Res 1974; 76: 185-212.

42. Mallol J, Hibbert ME, Robertson CF, Olinski A, Phelan PD. Inherent variability of pulmonary function tests in infants with bronchiolitis. Pediatr Pulmonol 1988; 5: 152-157.

43. Beardsmore CS, MacFadyen UM, Moosavi SS, Wimpress SP, Thompson J, Simpson H. Measurement of lung volumes during active and quiet sleep in infants. Pediatr Pulmonol 1989; 7: 71-77. 